\title{
Variation of Aulacoseira Granulata As An Eco-Pollution Indicator In Sub- Tropical Large River Ganga In India: A Multivariate Analytical Approach
}

Trupti Rani Mohanty

CIFRI: Central Inland Fisheries Research Institute

Nitish Kumar Tiwari

CIFRI: Central Inland Fisheries Research Institute

Suman Kumari

CIFRI: Central Inland Fisheries Research Institute

Archisman Ray

CIFRI: Central Inland Fisheries Research Institute

\section{Ranjan Kumar Manna}

CIFRI: Central Inland Fisheries Research Institute

\section{Supriti Bayen}

CIFRI: Central Inland Fisheries Research Institute

\section{Shreya Roy}

CIFRI: Central Inland Fisheries Research Institute Subhadeep Das Gupta

CIFRI: Central Inland Fisheries Research Institute Mitesh Hiradas Ramteke

CIFRI: Central Inland Fisheries Research Institute Himanshu Sekhar Swain

CIFRI: Central Inland Fisheries Research Institute Manisha Bhor

CIFRI: Central Inland Fisheries Research Institute

Basanta Kumar Das ( $\sim$ basantakumard@gmail.com)

Central Inland Fisheries Research Institute

\section{Research Article}

Keywords: Plankton bloom, Ganga, Ecological indicator, Aulacoseira granulata, WPI, PCA

Posted Date: August 30th, 2021

DOI: https://doi.org/10.21203/rs.3.rs-797085/v1

License: (1) This work is licensed under a Creative Commons Attribution 4.0 International License. Read Full License 


\begin{abstract}
Aulacoseira granulata (Ehrenberg) Simonsen 1979 is one of the major ecological indicators of the water quality of lotic as well as lentic aquatic ecosystems. To assess major environmental factors contributor to A.granulata bloom in the riverine system, a study was carried out from 2018 to 2019 comprising four different seasons at 11 sampling sites of river Ganga in the middle and lower stretch of river Ganga comprising freshwater and estuarine zones. For the analysis, different univariate, as well as multivariate analytical tools such as Principal Component Analysis (PCA) and Water pollution Index (WPI), were used. In the finding, it was observed that the average abundance of Aulacoseira granulata was found maximum during the winter season. Among all the studied sites, the maximum average abundance was at Balagarh (71576 cell $\mathrm{I}^{-1}$ ) and minimum at Diamond Harbour $\left(68\right.$ cell I $\left.^{-1}\right)$. The environmental factors such as dissolved oxygen, depth, and altitude showed a positive and water temperature negatively influencing the growth rate of $A$. granulate. WPI showed a significantly negative correlation with cell length. Finally, the study concludes that the blooming of Aulacoseira granulata is highly influenced by varied environmental conditions along the river Ganga suggesting possible eutrophication. Therefore, a certain minimum flow and depth especially during the lean season have to be maintained for the sustenance of planktonic biota in the river Ganga.
\end{abstract}

\title{
Introduction
}

Planktonic organisms are considered as a true biotic indicator of any sort of alterations in aquatic health. Being the nodal point of any aquatic food chain, it certainly depicts the condition through which an aquatic environment is passing by, as they are highly sensitive to any sort of abiotic abnormalities in the environment (Pham et al., 2017). The planktonic bloom in several open water aquatic systems has now become a serious concern globally which is further coupled with increasing anthropogenic pollution (Nwankwegu et al., 2019). Blooms are often a noticeable occurrence irrespective of the form of the aquatic ecosystem (lotic or lentic). Epiphytic plankton, mainly diatoms are being used as ecological indicators for several decades (Rimet et al., 2015). In a lotic ecosystem such as rivers and streams, diatoms form one of the ubiquitous planktonic communities (Jia et al., 2009). These, diatoms (Bacillariophyceae) have also been reported from the mighty Ganga river system in several past literatures (Pandey et al., 2017 \& Srivastava et al., 2020). Diatom community representing the genus Aulacoseira (commonly known as pennate diatoms) belonging to the phylum Bacillariophyceae are widely available species in various inland freshwater systems (Wang et al., 2009). They are found mostly in colonial growth habitats. The growth rate of the diatoms is faster in contrast to other phytoplanktonic taxa (Wetz\& Wheeler, 2007). The species representing the genus Aulacoseira form a linear colony by linking together with spines (Cox, 1996). They are very large, complex, oldest non-marine, and widely distributed diatoms (Ambawani et al., 2003) and best adapted to all inland water bodies like ponds, lakes, streams, and rivers. The genus Aulacoseira has been reported to have 144 species from all over the world (Guiry\&Guiry,2021). It is also reported to indicate a eutrophic condition in rivers as they accumulate to form high biomass (Hotzel \& Croome, 1996; Yang et al., 1997). Physical and chemical characteristics of an aquatic body like $\mathrm{pH}$, temperature, altitude, etc. play a vital role in the diversification of diatoms (You et al., 2009). Though very widely spread, the diatom species Aulacoseira granulata is considered to be the most dominant in carbonate-rich eutrophic water bodies (Kilham and Kilham, 1975; Margalef, 1983). They are among the few bloom-forming diatom species (Aulacoseira sp., Cyclotella sp., Stephanodiscus sp. and Fragilaria sp.) in hypereutrophic freshwater environments (Ha et al., 2003). Earlier works have reflected dominancy of the phytoplankton group mainly by diatoms (Bacillariophyceae) on river Ganga contributing to 100.0,56.0, and 50\% in upper, middle, and lower zones respectively (Pathak et al., 2010). The formation of bloom, the impact of abundance and growth of A.granulata have been documented in several lentic as well as lotic water bodies from all over the world (Wang et al., 2017 and Wang et al., 2020) but the variation and quantified data on the abundance of $A$. granulata is lacking in large flowing river systems is yet to be addressed. River Ganga is designated to be the largest river in India sprawling over 26 percent of the total Indian geographical area. The river gorges over three different eco-regions of India and thus sustaining a large number of multifarious in terms of several aquatic micro-flora and fauna. In the river Ganga, the diversity of diatoms was high during summer but density was high during winter due to A.granulata (Roshith et al., 2018). In this context, an attempt has been made to document the seasonal occurrence and pattern of $A$.granulata with the aiming of assessing the onsets of blooming conditions, factors triggering its actuality in the river, and its impact on ecological aspects. The study will also quantify and evaluate the A.granulata act as a pollution indicator species in the large subtropical river of India.

\section{Material And Methods}

\subsection{Study sites}

River Ganga is the largest in India and the fifth-largest in the world. The river emerges out to be cool in the uplands in the north and warmer during its course of flow until it's finally confluence to form the biggest delta in the world before draining into the Bay of Bengal in the east. The sites covered were almost 1208 Kilometers and a total of 11 sampling stations comprising the Indian States of Bihar and West Bengal. The entire stretch has been divided into two zones based on their hydrological characteristics. Freshwater zones are mainly comprised of Buxar, Patna, Bhagalpur, Farakka, Jangipur, and Berhampore. Likewise, the estuarine stretch covers the regions Balagarh, Tribeni, Godakhali, Diamond Harbour, and Fraserganj. All the sites have been selected based on their physical nature of water (tidal, etc.) and altitude gradient (Fig. 01).

\subsection{Climatic condition}

The entire Ganga basin specifically the middle and lower stretches experiences a typical monsoon n climate extending from June to October. Although there are some differences in precipitation between the middle and lower Ganga, the annual amount of rainfall remains the same which is $1000 \mathrm{~mm}$ (Quincey, 2017). Warm and dry seasons extend from March to May however, winter starts from November and ends in February.

\subsection{Sample collection and identification}

Plankton samples were collected quarterly for one year(2018 to 2019) covering all four tropical seasons. Composite samples from the mid-channel of the river were collected manually against the water current using the typical country boat. To maintain uniformity, a total of 100 liters of plankton samples were 
collected at first from the subsurface depth of 0.5-1.0 meters using a water sampler from which one liter of the mixed representative sample was taken out. Common conical tow net (mesh size $20 \mu \mathrm{m}$ ) was used to serve the purpose. One Litre of Plankton sample was also collected without any filtration, fixed in Lugol iodine solution, and analyzed based on sedimentation method to check if all representative of the species is present. Plankton samples were collected in dry polyethylene containers using $4 \%$ buffered formalin as a preservative. The identification was done following the standard literature such as Moro et al. (1991), Kulikovskiy et al. (2016), and the International Code of Biotic Nomenclature (ICBN). For valid and updated names AlgaeBase was followed. Samples from rich eutrophic regions were diluted for analysis. For taxonomic identification and qualitative assessment $0.5 \mathrm{mlof}$ each mixed sample was analyzed under the microscope (Model No.-Zeiss scopeA1). The counts were recorded in cell $\mathrm{I}^{-1}$. Cell size was represented by cell diameter and cell length; filament size was represented by filament width (cell diameter), filament length (in terms of cell numbers per filament).

\subsection{Waterquality parameter}

Water samples were collected between $0830 \mathrm{Hrs}$ to $0930 \mathrm{hrs}$ for determining various water quality parameters. In-situ analysis of parameters like water temperature $\left({ }^{\circ} \mathrm{C}\right), \mathrm{pH}$, conductivity $(\mu \mathrm{S} / \mathrm{cm})$, and turbidity (NTU) was carried out in the field by using YSI- Pro DSS multi-parameter probe, velocity (m/sec) was measured using a digital flow meter. Depth was measured using an ultrasonic depth meter. Transparency (cm) was measured by a Secchi disc. Dissolved oxygen (ppm) was done by Winkler's method in the field. Whereas for nutrient parameters such as Nitrate (ppm), Phosphate (ppm), Sulphate (ppm), and Silicate (ppm), water samples were taken to the laboratory at $4^{0} \mathrm{C}$. All the water quality parameters were analyzed following APHA, 2017.

\subsection{Water Pollution Index (WPI)}

For evaluation and characterization of the different sampling stations based on their chemical health, the Water Pollution Index was calculated, which has been derived and modified from the Nutrient Pollution Index developed in the United States by Dodds et al. (1998) and in South Korea by Lee and AN (2009) and lastly modified by Atique and An (2019). For the analysis of the nutrient regime total nitrogen, total phosphorus, ratio of total nitrogen: total phosphorus was utilized. For the analysis of the organic matter, Biochemical Oxygen Demand (BOD) was used. For ionic contents and solids, the total suspended solids and specific conductivity were used. Estimation of primary production was using Chlorophyll (Wang et al., 2017). The calculated values were compared and graded based on scores i.e. for Excellent (31-35), Good (25-29), Fair (19-23), poor (13-17), and for very poor (7-11).

\subsection{Statistical analysis}

Multivariate statistical analysis Principal Component Analysis (PCA) was followed using Past 4.03 software. So for the study 29 different environmental variables Phytoplankton (PHYTO); Aulacoseira granulata (AUL); Zooplankton (ZOO); Total plankton (TPLANK); Water temperature (WT); Depth (DEP); Transparency (TRAN); Velocity (VEL); Turbidity (TUR); Conductivity (COND); pH; Dissolved Oxygen (DO); Biochemical Oxygen Demand (BOD); Total Alkalinity (TALK) Free Carbon Dioxide ( $\mathrm{FCO}_{2}$ ), Calcium (CAL); Total Hardness (THAR); Salinity (SAL); Total Nitrogen (TOTN); Available Nitrogen (AVN); Total Phosphorus (TOTP); Silicate (SILI); Total Solid (TS); Total Dissolved Solid (TDS); Total Suspended Solid (TSS); Chlorophyll-A (CH-A); Chlorophyll-B (CH-B); Chlorophyll-C $(\mathrm{CH}-\mathrm{C})$ and Total Chlorophyll (TCHL) were used, which were distributed at 11 different sampling stations ( Fig. 04) i.e. Buxar, Patna, Bhagalpur, Farakka, Jangipur, Berhampore, Balagarh, Tribeni, Godakhali, Diamond Harbour and, Fraserganj.

Karl Pearson's correlation was also performed to find out the influencing factors which determinedly affect the bloom and growth of $A$.granulata using SPSS software 20.1 .

The Bray-Curtis cluster analysis was performed to know the similarity among the different stations based on total length, valve length, cell number, cell length, and density (Guinder et al., 2020).

\section{Results And Discussion}

\subsection{Morpho-taxonomic identification of $A$. granulata}

Class: Coscinodiscophyceae

Order: Aulacoseirales

Family: Aulacoseiraceae

A. granulata is a pinnate diatom belonging to the class Bacillariophyceae. The cells are tightly connected to form a long filament. The cell wall has rows of dots and a ring of spines, located at end of the cell. The deep valve mantle forms a right-angled junction with the valve face. Valve is striate. Chloroplasts are small disc or plate-like. The species is designated to be a nano-plankton $(<30 \mu \mathrm{m})$ comprising of series of valves. The average total length was found to be $418 \pm 2.89 \mu \mathrm{m}$ while the mean valve length was recorded to be $12.45 \pm 0.06 \mu \mathrm{m}$. The total length of the filament including spine length, valve length, total cell count, and cell length of a single filament varied significantly among all the stations. In the region (Behrampore, Balagarh, and Tribeni)where the density of $A$. granualata was more the size range was observed lower(Table 01). Picture of the A. granualata was given in Fig. 02 
Table 01

Morphological measurement of A. granualata (mean \pm )

\begin{tabular}{|lllll|}
\hline Sampling Stations & Avg. Total length $(\mu \mathrm{m})$ & Avg. Valve length $(\mu \mathrm{m})$ & Avg. Cell No. per filament & Avg. Cell length $(\mu \mathrm{m})$ \\
\hline Buxar & $950.37 \pm 27.32$ & $14.95 \pm 0.94$ & $16.78 \pm 0.45$ & $57.24 \pm 0.68$ \\
\hline Patna & $762.96 \pm 18.26$ & $13.29 \pm 0.51$ & $13.44 \pm 0.39$ & $54.70 \pm 1.28$ \\
\hline Bhagalpur & $635.78 \pm 20.21$ & $14.94 \pm 1.15$ & $12.00 \pm 0.32$ & $53.11 \pm 1.17$ \\
\hline Farakka & $344.56 \pm 6.49$ & $11.08 \pm 0.62$ & $11.00 \pm 0.42$ & $42.86 \pm 2.03$ \\
\hline Jangipur & $267.36 \pm 1.71$ & $9.58 \pm 0.62$ & $9.56 \pm 0.19$ & $29.12 \pm 0.51$ \\
\hline Behrampore & $361.17 \pm 2.54$ & $13.14 \pm 0.57$ & $11.78 \pm 0.42$ & $36.91 \pm 1.55$ \\
\hline Balagarh & $472.38 \pm 7.69$ & $10.57 \pm 0.39$ & $14.00 \pm 0.39$ & $36.69 \pm 0.87$ \\
\hline Tribeni & $210.10 \pm 1.89$ & $13.01 \pm 0.67$ & $8.44 \pm 0.21$ & $27.46 \pm 0.68$ \\
\hline Godakhali & $303.57 \pm 1.45$ & $12.91 \pm 0.45$ & $9.78 \pm 0.21$ & $31.59 \pm 0.55$ \\
\hline Diamond Harbour & $177.85 \pm 0.60$ & $11.23 \pm 0.55$ & $7.33 \pm 0.30$ & $26.07 \pm 0.77$ \\
\hline Fraserganj & $111.96 \pm 6.38$ & $12.23 \pm 0.66$ & $6.33 \pm 0.47$ & $29.52 \pm 1.10$ \\
\hline
\end{tabular}

3.2.Seasonal abundance of A. granulata

It is noteworthy that the total volume of the plankton varies seasonally in all aquatic systems and the same has been observed in the present study of the river Ganga (Lakshminarayana, 1965). During the pre-monsoon months (February to April), the density of the A. granulata was observed 905 cell I-1. The count of A. granulata was recorded lowest $\left(113 \mathrm{cell} \mathrm{I}^{-1}\right)$ during the monsoon months (June-August). This can be attributed to the huge influx of water in the Ganga from adjacent tributaries during peak monsoon. This is characterized by high water velocity (Avg. $0.6 \mathrm{~m} \mathrm{sec}^{-1}$ ), depth (Av. $10.1 \mathrm{~m}$ ) resulting in the breaking of the thermocline. During post-monsoon (September to October) the density of the A. granulata was observed at 352 cell ${ }^{-1}$. The riverine diatom, A.granulata dominated during the winter season extending from December to January with the abundance of 52970 cells $I^{-1}$ through throughout the entire river stretch (Fig. 03). This might be due to the sluggish nature of water velocity (Av.0.4 m sec${ }^{-1}$ ) and conducive environmental factors during the period.

\subsection{Stretch wise distribution of A.granulata abundance}

The sudden prolific rise of diatom A. granulate has been recorded from all the stations with the highest value at Balagarh ( 285863 cell $\left.I^{-1}\right)$ followed by Tribeni (153608 cell $\mathrm{I}^{-1}$ ) and Behrampore (20942 cell $\mathrm{I}^{-1}$ ). The blooming conditions of $A$.granulata have been recorded to significantly peak during the winter months. During post-monsoon months, the highest abundance of A.granulata was found at Buxar and Tribeni ( 5652 cell $I^{-1}$ ) while correspondingly lowest abundance was recorded at the estuarine stretch (Godakhali. Diamond Harbour, and Fraserganj). All the stations showed the lowest dominance of $A$.granulata throughout during monsoon. In this season highest dominance of the diatom was observed at Bhagalpur (780 cell liter ${ }^{-1}$ ) while the least dominance was observed at Buxar and Diamond Harbour. The diatom A.granulata was recorded high at Bhagalpur (5652 cell I-1) and low at Behrampore during pre-monsoon. Stretch wise seasonal variation of $A$.granulata has been shown in Fig. 4.

\subsection{Water Pollution Index (WPI)}

The Water Pollution Index (WPI) was calculated for the assessment of the different sampling stations (Table 02). The analysis revealed that all the sampling stations were in the fair category of WPI. Although the scores varied among the different stations between 19 to 23 . The score of 19 was obtained from different stretch viz. Buxar, Patna, Bhagalpur, Balagarh, and Fraserganj. The score value of 21 was obtained at Farakka, Tribeni, and Diamond Harbour whereas, comparatively higher scores of 23 were obtained at Jangipur, Behrampore, and Godakhali. 
Table 02

Water Pollution Index of the sampling sites

\begin{tabular}{|c|c|c|c|c|c|c|c|c|c|c|c|c|c|}
\hline \multirow[t]{2}{*}{ Category } & \multirow{2}{*}{$\begin{array}{l}\text { Model } \\
\text { metrics (M) }\end{array}$} & \multicolumn{3}{|c|}{ Scoring criteria } & \multicolumn{9}{|c|}{ Mean \pm standard error scores } \\
\hline & & 5 & 3 & 1 & Buxar & Patna & Bhagalpur & Farakka & Jangipur & Behrampore & Balagarh & Tribeni & Godal \\
\hline \multirow[t]{8}{*}{$\begin{array}{l}\text { Nutrient } \\
\text { Regime }\end{array}$} & \multirow[t]{2}{*}{$\begin{array}{l}\text { M1 : Total } \\
\text { Nitrogen } \\
\text { (mg/L) }\end{array}$} & \multirow[t]{2}{*}{$<$} & \multirow[t]{2}{*}{$\begin{array}{l}1.5- \\
3.0\end{array}$} & \multirow[t]{2}{*}{$>3$} & $\begin{array}{l}0.80 \pm \\
0.06\end{array}$ & $\begin{array}{l}0.85 \pm \\
0.09\end{array}$ & $\begin{array}{l}1.26 \\
\pm 0.09\end{array}$ & $\begin{array}{l}0.44 \pm \\
0.11\end{array}$ & $\begin{array}{l}0.67 \pm \\
0.11\end{array}$ & \multirow{2}{*}{$\begin{array}{l}0.68 \pm 0.14 \\
\text { (5) }\end{array}$} & \multirow{2}{*}{$\begin{array}{l}0.47 \pm \\
0.04 \\
(5)\end{array}$} & \multirow{2}{*}{$\begin{array}{l}0.59 \pm \\
0.08 \\
(5)\end{array}$} & \multirow{2}{*}{$\begin{array}{l}0.76 \pm \\
0.08 \\
(5)\end{array}$} \\
\hline & & & & & (5) & $(5)$ & (5) & (5) & (5) & & & & \\
\hline & \multirow[t]{2}{*}{$\begin{array}{l}\text { M2 : Total } \\
\text { Phosphorus } \\
(\mu \mathrm{g} / \mathrm{L}\end{array}$} & \multirow[t]{2}{*}{$<$} & \multirow[t]{2}{*}{$\begin{array}{l}30- \\
100\end{array}$} & \multirow[t]{2}{*}{$>100$} & \multirow{2}{*}{$\begin{array}{l}0.24 \pm \\
0.04 \\
(5)\end{array}$} & 0.19 & \multirow{2}{*}{$\begin{array}{l}0.40 \\
\pm 0.12 \\
(5)\end{array}$} & \multirow{2}{*}{$\begin{array}{l}0.21 \pm \\
0.05 \\
(5)\end{array}$} & \multirow{2}{*}{$\begin{array}{l}0.07 \pm \\
0.01 \\
(5)\end{array}$} & \multirow{2}{*}{$\begin{array}{l}0.11 \pm 0.03 \\
(5)\end{array}$} & \multirow{2}{*}{$\begin{array}{l}0.07 \pm \\
0.01 \\
(5)\end{array}$} & \multirow{2}{*}{$\begin{array}{l}0.07 \pm \\
0.01 \\
(5)\end{array}$} & \multirow[t]{2}{*}{$\begin{array}{l}0.22 \pm \\
0.06(\end{array}$} \\
\hline & & & & & & $(5)$ & & & & & & & \\
\hline & \multirow[t]{4}{*}{$\begin{array}{l}\text { M3 : TN:TP } \\
\text { ratio }\end{array}$} & \multirow[t]{4}{*}{$\begin{array}{l}> \\
50\end{array}$} & \multirow[t]{4}{*}{$\begin{array}{l}20- \\
100\end{array}$} & \multirow[t]{4}{*}{$\begin{array}{l}< \\
20\end{array}$} & & $\begin{array}{l}6.31 \pm \\
1.16\end{array}$ & \multirow{4}{*}{$\begin{array}{l}6.66 \\
\pm 1.28 \\
(1)\end{array}$} & \multirow{4}{*}{$\begin{array}{l}8.67 \pm \\
2.17 \\
(1)\end{array}$} & \multirow{4}{*}{$\begin{array}{l}13.90 \pm \\
3.15 \\
(1)\end{array}$} & $7.16 \pm 0.39$ & $\begin{array}{l}7.54 \pm \\
0.75\end{array}$ & & $\begin{array}{l}13.05 \\
3.46\end{array}$ \\
\hline & & & & & & (1) & & & & (1) & (1) & $\pm 2 . / 5$ & \\
\hline & & & & & 1.02 & & & & & & & (1) & \\
\hline & & & & & (1) & & & & & & & & \\
\hline $\begin{array}{l}\text { Organic } \\
\text { Matter }\end{array}$ & $\begin{array}{l}\text { M4 : BOD } \\
(\mathrm{mg} / \mathrm{L})\end{array}$ & $<1$ & $1-2.5$ & 2.5 & $1.56 \pm$ & $\begin{array}{l}1.68 \pm \\
0.22\end{array}$ & $\begin{array}{l}1.22 \\
\pm 0.16\end{array}$ & $\begin{array}{l}1.22 \pm \\
0.30\end{array}$ & $\begin{array}{l}0.76 \pm \\
0.06\end{array}$ & $0.73 \pm 0.07$ & $\begin{array}{l}1.25 \pm \\
0.05\end{array}$ & $\begin{array}{l}0.98 \pm \\
0.12\end{array}$ & $\begin{array}{l}0.93 \pm \\
0.13(\end{array}$ \\
\hline & & & & & $\begin{array}{l}0.11 \\
(3)\end{array}$ & (3) & (3) & (3) & (5) & (5) & (3) & & \\
\hline $\begin{array}{l}\text { lonic } \\
\text { Contents }\end{array}$ & $\begin{array}{l}\text { M5: TSS } \\
(\mathrm{mg} / \mathrm{L})\end{array}$ & $<4$ & $4-10$ & $>$ & $\begin{array}{l}157.15 \\
\pm 48.25\end{array}$ & $\begin{array}{l}87.60 \pm \\
23.71\end{array}$ & $\begin{array}{l}36.10 \\
\pm\end{array}$ & $\begin{array}{l}96.30 \pm \\
24.08\end{array}$ & $\begin{array}{l}72.10 \pm \\
28.17\end{array}$ & $\begin{array}{l}69.50 \pm \\
17.28\end{array}$ & 89.40 & $\begin{array}{l}24.10 \\
\pm 5.61\end{array}$ & $\begin{array}{l}161.0 \\
30.67\end{array}$ \\
\hline ana Sollas & & & & & (1) & (1) & $\begin{array}{l}15.08 \\
(1)\end{array}$ & (1) & (1) & (1) & $\begin{array}{l} \pm 26.18 \\
(1)\end{array}$ & (1) & (1) \\
\hline & $\begin{array}{l}\text { M6 : Sp. } \\
\text { Conductivity }\end{array}$ & $<$ & $\begin{array}{l}180- \\
300\end{array}$ & 300 & $\begin{array}{l}500.50 \\
\pm\end{array}$ & $\begin{array}{l}368 \pm \\
33.01\end{array}$ & $\begin{array}{l}484 \pm \\
46.27\end{array}$ & $\begin{array}{l}362.25 \\
\pm 90.56\end{array}$ & $\begin{array}{l}320.5 \pm \\
31.18\end{array}$ & $\begin{array}{l}331.75 \pm \\
22.08\end{array}$ & 319.25 & $\begin{array}{l}301 \pm \\
28.03\end{array}$ & $\begin{array}{l}366.5 \\
11.74\end{array}$ \\
\hline & /cm & & & & 22.61 & (1) & (1) & (1) & (1) & (1) & \pm 30.55 & (1) & (1) \\
\hline & & & & & (1) & & & & & & (1) & & \\
\hline $\begin{array}{l}\text { Primary } \\
\text { Production }\end{array}$ & $\begin{array}{l}\text { M7 : } \\
\text { Chlorophyll }\end{array}$ & $<3$ & $3-10$ & $>$ & $\begin{array}{l}4.05 \pm \\
0.89\end{array}$ & $\begin{array}{l}4.58 \pm \\
0.75\end{array}$ & $\begin{array}{l}5.24 \\
\pm 1.24\end{array}$ & $\begin{array}{l}2.55 \pm \\
0.64\end{array}$ & $\begin{array}{l}1.88 \pm \\
0.28(5)\end{array}$ & $2.82 \pm 0.77$ & $\begin{array}{l}5.77 \pm \\
1.48\end{array}$ & $\begin{array}{l}4.33 \pm \\
0.85\end{array}$ & $\begin{array}{l}3.19 \pm \\
0.55\end{array}$ \\
\hline mivilut & (1) & & & & (3) & (3) & (3) & (5) & & & (3) & (3) & (5) \\
\hline & $\begin{array}{l}\text { Final Scores } \\
\text { (Model } \\
\text { Criteria of } \\
\text { WPI) }\end{array}$ & & & & 19 & 19 & 19 & 21 & 23 & 23 & 19 & 21 & 23 \\
\hline & $\begin{array}{l}\text { Water } \\
\text { Quality } \\
\text { Criteria }\end{array}$ & & & & fair & fair & fair & fair & fair & fair & fair & fair & fair \\
\hline
\end{tabular}




\begin{tabular}{|c|c|c|c|c|c|c|c|c|c|c|c|c|c|c|c|}
\hline & Aul & WT & DEP & TRAN & VEL & TUR & COND & $\mathrm{pH}$ & DO & BOD & TALK & $\mathrm{FCO} 2$ & CAL & THAR & $S$ \\
\hline Aul & 1 & & & & & & & & & & & & & & \\
\hline WT & $-.402^{\star \star}$ & 1 & & & & & & & & & & & & & \\
\hline DEP & $.342^{*}$ & -.075 & 1 & & & & & & & & & & & & \\
\hline TRAN & -.081 & -.232 & $-.327^{*}$ & 1 & & & & & & & & & & & \\
\hline VEL & .110 & .168 & $.302^{\star}$ & $-.459^{\star \star}$ & 1 & & & & & & & & & & \\
\hline TUR & -.126 & $.350^{\star}$ & .248 & $-.411^{\star \star}$ & $.319^{\star}$ & 1 & & & & & & & & & \\
\hline COND & -.094 & .087 & -.020 & -.109 & -.083 & .052 & 1 & & & & & & & & \\
\hline $\mathrm{pH}$ & .144 & $-.447^{\star *}$ & -.101 & .005 & .205 & -.241 & -.121 & 1 & & & & & & & \\
\hline DO & $.507^{\star *}$ & $-.599^{\star *}$ & -.018 & .176 & -.103 & $-.488^{\star *}$ & $-.427^{\star *}$ & $.477^{\star \star}$ & 1 & & & & & & \\
\hline BOD & -.120 & -.141 & .016 & .118 & -.116 & -.027 & $.354^{*}$ & -.221 & -.078 & 1 & & & & & \\
\hline TALK & .114 & $-.586^{\star *}$ & .067 & $.331^{*}$ & $-.355^{\star}$ & -.262 & .076 & .093 & $.345^{\star}$ & .269 & 1 & & & & \\
\hline FCO2 & -.166 & .214 & .037 & -.082 & -.002 & $.305^{*}$ & -.060 & -.199 & -.103 & -.088 & -.068 & 1 & & & \\
\hline CAL & -.011 & .067 & -.107 & -.081 & -.040 & .086 & $.743^{* *}$ & .067 & -.285 & -.109 & .084 & -.208 & 1 & & \\
\hline THAR & -.094 & .139 & -.075 & -.133 & -.064 & .066 & $.898^{* \star}$ & -.081 & $-.369^{*}$ & .135 & .046 & -.108 & $.901^{\star \star}$ & 1 & \\
\hline SAL & -.100 & .104 & -.006 & -.129 & -.072 & .078 & $.995^{\star \star}$ & -.128 & $-.442^{\star \star}$ & $.318^{\star}$ & .080 & -.074 & $.790^{\star \star}$ & $.930^{\star \star}$ & 1 \\
\hline TOTN & -.133 & .013 & -.112 & .214 & -.117 & $.375^{\star}$ & -.187 & -.090 & -.111 & -.015 & .213 & $.493^{\star \star}$ & -.167 & -.230 & - \\
\hline AVN & .056 & .042 & -.239 & .198 & -.124 & -.218 & -.234 & .031 & $.327^{\star}$ & .005 & .022 & -.097 & -.172 & -.177 & - \\
\hline TOTP & -.141 & .030 & -.177 & $.579^{\star \star}$ & $-.373^{\star}$ & -.049 & -.077 & -.178 & -.002 & .104 & .210 & .278 & -.157 & -.102 & - \\
\hline SILI & .135 & -.081 & -.213 & $.530^{\star \star}$ & -.215 & -.237 & $-.441^{\star *}$ & .118 & $.366^{*}$ & .018 & .069 & .012 & $-.307^{*}$ & $-.394^{\star *}$ & - \\
\hline TS & -.098 & .123 & .065 & -.166 & .068 & $.329^{\star}$ & $.738^{\star \star}$ & -.085 & $-.487^{\star *}$ & .194 & -.039 & -.079 & $.726^{\star \star}$ & $.740^{* *}$ & .7 \\
\hline TDS & -.098 & .099 & .114 & -.175 & .067 & $.323^{\star}$ & $.695^{\star *}$ & -.089 & $-.442^{\star *}$ & .233 & -.018 & -.075 & $.666^{\star *}$ & $.718^{\star *}$ & .7 \\
\hline TSS & -.073 & .063 & -.001 & -.078 & -.017 & .152 & $.765^{\star \star}$ & -.028 & $-.434^{\star *}$ & .149 & .148 & -.102 & $.699^{\star \star}$ & $.617^{\star *}$ & .7 \\
\hline $\mathrm{CHA}$ & $.564^{\star \star}$ & $-.343^{*}$ & .135 & -.014 & -.023 & -.116 & -.014 & .223 & $.380^{\star}$ & -.001 & .215 & .207 & -.030 & -.100 & - \\
\hline $\mathrm{CHB}$ & -.033 & .175 & .148 & -.088 & -.060 & $.358^{*}$ & $.429^{\star *}$ & -.237 & $-.369^{\star}$ & .206 & -.053 & .300 & .161 & .247 & .4 \\
\hline $\mathrm{CHC}$ & -.081 & .210 & .218 & -.221 & .070 & $.407^{\star *}$ & $.526^{\star *}$ & -.236 & $-.524^{\star *}$ & .193 & -.163 & .280 & .231 & $.324^{*}$ & .5 \\
\hline TCHL & .264 & -.072 & .228 & -.145 & .054 & .241 & $.346^{*}$ & -.080 & -.174 & .135 & .060 & .230 & .139 & .165 & .3 \\
\hline
\end{tabular}

Table 04

Eigenvalues and variance $\%$ of the

\begin{tabular}{|lll|}
\hline PC & Eigenvalue & \% variance \\
\hline 1 & 7.23501 & 28.94 \\
\hline 2 & 3.31685 & 13.267 \\
\hline 3 & 3.0954 & 12.382 \\
\hline 4 & 2.38506 & 9.5402 \\
\hline 5 & 1.37163 & 5.4865 \\
\hline 6 & 1.23455 & 4.9382 \\
\hline 7 & 1.07258 & 4.2903 \\
\hline
\end{tabular}


Table 05

Principal components of 7 Extracted PC'S having Eigenvalues $>1$

\begin{tabular}{|lccccccc|}
\hline & PC 1 & PC 2 & PC 3 & PC 4 & PC 5 & PC 6 & PC 7 \\
\hline Aul & -0.16 & -0.13 & 0.35 & 0.65 & 0.14 & 0.29 & 0.24 \\
\hline WT & 0.26 & 0.45 & -0.5 & -0.39 & 0.22 & 0.29 & -0.03 \\
\hline DEP & 0.12 & 0.27 & 0.02 & 0.54 & -0.35 & 0.09 & 0.41 \\
\hline TRAN & -0.3 & -0.3 & 0.44 & -0.56 & 0.01 & -0.03 & -0.01 \\
\hline VEL & 0.08 & 0.27 & -0.41 & 0.51 & 0.15 & 0.02 & 0.25 \\
\hline TUR & 0.38 & 0.62 & -0.18 & 0.02 & 0.08 & -0.23 & 0.43 \\
\hline COND & 0.89 & -0.29 & 0.14 & -0.07 & -0.03 & 0.1 & -0.09 \\
\hline PH & -0.22 & -0.37 & 0.07 & 0.42 & 0.39 & -0.29 & -0.11 \\
\hline DO & -0.65 & -0.38 & 0.32 & 0.37 & 0.07 & 0.01 & 0.03 \\
\hline BOD & 0.22 & -0.11 & 0.28 & -0.2 & -0.65 & 0.2 & 0.18 \\
\hline TALK & -0.08 & -0.39 & 0.57 & -0.02 & -0.35 & -0.4 & 0.15 \\
\hline FCO2 & 0.02 & 0.6 & 0.21 & -0.18 & 0.17 & -0.27 & 0.01 \\
\hline CAL & 0.74 & -0.45 & -0.02 & 0 & 0.36 & -0.11 & 0.02 \\
\hline THAR & 0.83 & -0.4 & -0.02 & -0.09 & 0.15 & 0.03 & -0.01 \\
\hline SAL & 0.91 & -0.31 & 0.1 & -0.07 & -0.01 & 0.08 & -0.06 \\
\hline TOTN & -0.1 & 0.48 & 0.37 & -0.3 & 0.12 & -0.55 & 0.14 \\
\hline AVN & -0.44 & -0.25 & -0.04 & -0.32 & 0.22 & 0.4 & 0.31 \\
\hline TOTP & -0.15 & 0.12 & 0.54 & -0.58 & 0.17 & 0.11 & 0.23 \\
\hline SILI & -0.56 & -0.05 & 0.34 & -0.34 & 0.3 & 0.28 & 0.28 \\
\hline TS & 0.86 & -0.2 & -0.03 & -0.04 & 0.1 & -0.06 & 0.3 \\
\hline TDS & 0.81 & -0.21 & -0.06 & -0.03 & 0.04 & -0.07 & 0.37 \\
\hline TSS & 0.78 & -0.23 & 0.12 & -0.02 & 0.08 & -0.11 & 0 \\
\hline CH-A & -0.05 & 0.12 & 0.72 & 0.45 & 0.25 & 0.08 & -0.06 \\
\hline CH-B & 0.56 & 0.49 & 0.44 & -0.03 & -0.02 & 0.18 & -0.18 \\
\hline & 0.69 & 0.5 & 0.3 & 0.07 & -0.05 & 0.17 & -0.23 \\
\hline & 0.44 & 0.42 & 0.65 & 0.27 & 0.1 & 0.18 & -0.13 \\
\hline
\end{tabular}

The correlation was done between WPI and several other morphological characteristics (total length of the filament, valve length, total cell count of one filament, cell length of one filament, and abundance) of A.granulata. The same correlation was obtained between different structural parameters and a significantly negative correlation with WPI was obtained with Cell length $(r=-0.663)$ (Fig. 03). Cell total length $(r=-0.587, p>0.01)$, Valve length $(r=-0.492, p$ $>0.01)$, cell number $(r=-0.518, p>0.01)$ and cell density were found to negatively correlate with WPI but not significantly. It indicated when the value of WPI was high the total length of the cell decreases.

\subsection{Correlation between different water quality variables and A.granulataabundance}

The Karl Pearson correlation was carried out in which the significant correlation $(p<0.05)$ was observed with different environmental constraints. i.e. Water temperature (WT), Depth (DEP), Transparency (TRAN), Velocity ( VEL), Turbidity ( TUR), Conductivity ( COND), pH, Dissolved Oxygen ( DO), Biochemical Oxygen Demand (BOD), Total Alkalinity ( TALK), Free Carbon dioxide ( FCO2), Calcium ( CAL) Total Hardness ( THAR), Salinity ( SAL), Total Nitrogen ( TOTN), Available Nitrogen (AN), Total Phos ( TOTP), Silicate ( SILI), Total Solid ( TS), Total Dissolved Solids ( TDS), Total Suspended Solids ( TSS), Chlorophyll - A ( $\mathrm{CHA})$, Chlorophyll - B ( CHB), Chlorophyll- $\mathrm{C}(\mathrm{CHC})$ and Total Chlorophyll (TCHL). Table 3.which can be considered as influencing factors that determinedly affect the bloom and growth of A.granulata. Significantly positive correlation was observed with Depth $(r=0.342)$, dissolved $0 x y g e n(r=0.507)$, and Chlorophyll A $(r=0.564)$. However, a significantly negative correlation was observed with water temperature $(r=-0.402)$.

\subsection{Principal Component Analysis}

In the study, 7 PCs were selected where the eigenvalue was $>1$. The combined variance percentage for the 7 PCs was $78.84 \%$. In the 1 st PC where the variance percentage was $28.94 \%$, the strong positive loading was observed with conductivity $(r=0.89)$, total hardness $(r=0.83)$, salinity ( $r=0.91)$ and total suspended solids ( $r=0.78)$, while moderate positive loading was observed with calcium $(r=0.74)$, chlorophyll $B(r=0.56)$, and chlorophyll $C(r=0.69)$. No strong negative loading was observed. Moderate negative loading was found with dissolved oxygen $(r=-0.65)$ and silicate $(r=-0.56)$. 2nd PC having $13.27 \%$ variance has no 
strong positive loading. Moderate positive loading was observed with Free Carbon dioxide $(r=0.60)$ and chlorophyll-C $(r=0.50)$, While there was no strong and moderate negative loading observed in the 2nd PC. In the 3rd PC, the variance percentage was (12.39\%) having moderate positive loading was observed with total alkalinity $(r=0.57)$ and total chlorophyll $(r=0.65)$. Moderate negative loading was observed with water temperature $(r=-0.50)$, in the 4 th PC, the variance of $9.54 \%$ was observed. Having moderate positive loading with Aulacoseria granulate $(r=0.65)$ and Depth $(r=0.54)$ while moderate negative loading was observed with transparency $(r=-0.56)$ and Total Phosphorus $(r=-0.58)$. In 5th PC the variance \% was $(5.49 \%)$ whereas no strong and moderate positive loading was observed. Moderate negative loading was found with Biochemical Oxygen Demand ( $r=-0.65)$. In 6 th Pc, the variance \% was $4.94 \%$, and in this, there was no strong and moderate positive loading was observed. In the case of nutrient parameters, moderate negative loading was found with total nitrogen ( $r=-0.55)$. In 7th Pc where the variance \% was 4.30 and there were no strong and moderate positive or negative loading observed in it. The result of PCA was given in Fig .06 and Table no. 04 and 05

\subsection{Cluster analysis}

In the analysis, it was found that cophenetic correlation was 0.92 which shows the best suitability of the data for cluster analysis among the different stations. In the analysis 2 paired groups were formed. In the first paired group the similarity was found between Bhagalpur, Behrampore, Tribeni, and Balagarh. The 2nd group has 3 subgroups. Firstly, Patna and Buxar showed maximum similarity. Stations likeFarakka and Godakhali showed a similar trend in the second subgroup. The same trend was followed in the third subgroup between diamond Harbour and Fraserganj(Fig. 07).

\section{WPI vis-à-vis morphological variation}

For comparing the morphological traits by the water pollution index (WPI), we relied upon Karl Pearson correlation methods. The data highlighted that all the morphological traits exhibited an inverse relationship with WPI. Interestingly, the relationship between cell length and WPI indicated significant negative correlation $(r=-0.66, p>0.01)$. The mean cell number per filament was also recorded relatively consistent with $10.94 \pm 3.08$. This can be attributed to that WPI influences the overall morpho characteristics of the diatom. The results outlined that, total cell length of the diatoms was observed to decrease significantly with the increased value of WPI. Further, the findings suggest a significant variation in other diatom morphology with the chemical loadings. Total nitrogen showed a significant positive correlation with the valve length $(r=0.706, p>0.05)$. Similarly, Total phosphate revealed a significant positive relationship with valve length $(r=0.646, p>0.05)$ and cell length $(r=0.687, p>0.05)$ respectively. Silicate was also found to establish an intricate positive relationship with the morpho parameters like valve length $(r=0.711, p>0.05)$ and cell length $(r=0.640, p>0.05)$ respectively.

\section{Discussion}

Planktonic organisms are considered as the aquatic bioindicator in the open water system, and, especially in the riverine system (Parmar et al., 2016) and the blooming condition of any of the diatoms myself explain the eutrophic status of the water quality which signifies the lower water flow rate in the system (Yang et al., 2012). The study was conducted to monitor the blooming condition and distribution pattern of $A$. granulate in the Ganga River system.

\subsection{Taxonomy}

Bacillariophyceae is widely recognized as a diatom and are predominantly unicellular consisting of rigid box-shaped silicified cell walls recognized as frustules(Cupp., 1943). The species is first reported by Simonsen in 197 (M.D. \& Guiry,2021) whose Basionym is Gaillonella granulata (Ehrenberg) and the synonym is Melosira granulata (Ehrenberg) Ralfs (Mayama,1991). The morphological characteristics ensuring the confirmation of $A$. granulata consist of long linked cells with upright spines inserted on valves (Cox,1996). The variety is characterized by the presence of 1-4 separated pines (Bicudo et al., 2016), average total length was $418 \pm 2.89 \mu \mathrm{m}$; mean valve length was $12.45 \pm 0.06 \mu$ mcell length (Cox,1996). The species $A$. granulata exhibits a diverse range of morphological variations (Kilham et al., 1975). The structural variation of $A$. granulata in the different aquatic ecosystems has been observed diversely (Wang et al., 2020).

Ecology and distribution: This species has a worldwide distribution (Guiry\&Guiry 2014) and occurs in a wide range of trophic conditions, but is mostly associated with eutrophic waters (Taylor et al. 2007).

\subsection{Distribution and composition pattern}

Many genera and species of phytoplankton communities belong to the Bacillariophyceae have the potential to enhance the degree of eutrophication in the river Ganga (Srivastava et al., 2016). Similarly, Roshith et al. (2018) have also observed that diatom, A.granulata, dominant species among the phytoplankton population in the summer and winter seasons. Lakshminarayana (1995) also reported that the diatoms had two peak points one is summer season (March) and another one is winter (December) in the river Ganga. The present investigation also agreed with the findings that the species $A$.granulata has shown a higher degree of abundance during pre-monsoon ( $905 \mathrm{cell} \mathrm{I}^{-1}$ ) and winter season (53188 cell $\mathrm{I}^{-1}$ ). The sudden plentiful rise of diatom A. granulate has been confronted from all the stations with the highest at Balagarh (285863 cell $\mathrm{I}^{-1}$ ) followed by Tribeni $\left(153608 \mathrm{cell}^{-1}\right)$ and Behrampore $\left(20942 \mathrm{cell}^{-1}\right)$.

The Bray-Curtis cluster analysis was performed to know the similarity among the different stations based on total length, valve length, cell number, cell length, and density (Guinder et al.2020). The present study indicated a similar trend of the population in all the freshwater and saline water zones. The introduction of saline water in the lower sites like Fraserganj and Diamond Harbour has also created alteration in the morphological structure. The results obtained were found in agreement with previous studies (Blum et al. 1957; Kanaya and Kikuchi, 2008).

\subsection{Physico-chemical properties}


Among the different physicochemical parameters, the significantly influenceable parameters are water temperature $(r=-0.402)$, depth $(r=0.342)$, dissolved oxygen ( $r=0.507)$, and chlorophyll A ( $r=0.564)$, which was similar to the other studies of different water bodies such as Danjiangkou Reservoir, China ( Zheng et al., 2020). Water temperature negatively affected the growth of $A$. granulata showed a higher growth rate during the winter season. The different nutrient parameters are dependent on water temperature (Thomas et al., 2017), which also affects the chlorophyll A composition and variation in the river system, by which the planktonic diversity and density are dependent (Moeller et al., 2019). Thus composition and variation of chlorophyll control the growth of plankton communities (Moeller et al., 2019). Similar observations i.e. the influence of water temperature on plankton growth specifically freshwater diatoms were found in several studies (Zhang et al., 2019). Similarly, the density has been positively influenced by depth during winter, higher depths were observed in the river as similar to the Gharraf river of Iraq (Al-Husseini et al., 2020). A significant positive correlation with Dissolved oxygen was also observed, during the daytime with sunlight, the photosynthetic activities of the phytoplankton get enhanced, and relatively more oxygen is released in the aquatic system when the numbers of Phytoplankton are higher and is also depending on water temperature (Sekerci et al., 2018). The higher dissolved oxygen in the riverine system has also been reported during the post-monsoon and winter season. Chlorophyll A greatly influences the process of photosynthesis in aquatic plants (Tsai et al., 2019). Similar observations were also found in the present findings as a significantly positive correlation with chlorophyll A was found with $A$. granulata.

PCA bi-plot is a well-known statistical tool for the analysis of specific variable and their interdependency on the other environmental variables (Singh et al., 2019). The principal component analysis is a widely accepted multivariate statistical tool used for the analysis of the wide number of environmental factors affecting the diversity and density of the planktonic species (Pratiwi et al., 2019; Herawati et al., 2020 and Matta et al., 2020). In this finding as the 1st PC is having positive loading with conductivity, total hardness, salinity, total suspended solids, calcium, chlorophyll-B, and chlorophyll-C while the negative loading was found with dissolved oxygen and silicate. The loading revealed the influence of the estuarine zone of the river. The same has been observed in other studies made in the estuarine stretch (Hooghly estuary) of river Ganga by Rakshit et al.(2017). The 2nd PC is having positive loading with free $\mathrm{CO}_{2}$, and Chlorophyll $\mathrm{C}$ shows the impact of monsoon due to Cloudy weather the free $\mathrm{CO}_{2}$ and higher chlorophyll content were observed in the riverine system and the observations were the same as that of the Erai river of Maharashtra, India and in the metropolitan city of Korea (Shende et al.,2019, and Hong et al., 2020). The 3rd PC is having positive loading with total alkalinity, total chlorophyll while negative loading was observed with water temperature the PC may also have denoted the contribution of organic matter loading and ion dissolution in the riverine system. The observations were similar to that of the upper stretch of river Ganga (Dimri et al., 2020). The 4th PC where the positive loading was observed with A.granualata and depth and negative loading was found with transparency and total phosphorus. The PC signifies the importance and influence of river depth. The negative loading with water transparency signifies the relatively higher riverine velocity which causes turbidity in the river (Ayoade et al., 2009). The negative loading with total phosphorus also signifies the higher riverine flow in which the nutrients such as phosphorus and nitrogen get dissolved. Similar observations were also observed with the Mississippi river where the maximum blooms of cyanotoxin-producing plankton were observed (Bargu et al., 2011). In the 5th and 6th PC, the negative loading was observed with biochemical oxygen demand and total nitrogen. This signifies the pollution status of the river and higher nutrient load which may cause eutrophication in the riverine system.

The use of WPI for the zonation and chemical characterization of the riverine system has been widely used in the studies of the large river such as in the Shanchong river of China (Wang et al., 2015), Sava River, Serbia (Popovic et al., 2016) and Damodar River, India (Chakraborty et al., 2021). The score of the WPI index was varied between 19-23. depicting fair water conditions throughout the stretch. The different WPI scores such as 19 were obtained from Buxar, Patna, Bhagalpur, Balagarh, and Fraserganj, while the score value of 21 was obtained at Farakka, Tribeni, and Diamond Harbour. However, comparatively higher scores of 23 were yielded at Jangipur, Behrampore, and Godakhali. The higher values signify the better quality of water and vice-versa (Atique et al., 2019 \& 2020). As the structural differentiation of the Aulacoseira granulata has been used in many of the studies such as in the pearl river of China (Wang et al., 2017\& 2020) for the assessment of aquatic ecosystems. The correlation was done between WPI and total length of the filament, valve length, total cell count of one filament, and cell length of one filament and abundance. A significantly negative correlation $(r=-0.663)$ with WPI was obtained with cell length. It is very much evident that the parameters related to the quantitative structure are very much influenced by the environmental changes (Bedoshvili et al. 2007; Poister et al. 2012). In the present study, the overall cell length varied significantly with the computed value of WPI. On the other hand, chemical parameters showed an intricate relationship with the morphological parameters also. Jewson et al. 1992 has opined that these environmental parameters have a strong association with its growth.

As in the earlier studies, it was also found that the blooming condition of the diatoms like $A$. granulata signifies the deteriorated environmental condition of the riverine system which hinders the growth and survival of the native aquatic organisms (Joung et al., 2013 and Poister et al., 2015) by adversely affecting their ecological niche. In the present study also the deteriorated environment has been shown with many of the environmental variables and morphological traits of the $A$. granualta which shows the significant correlation among each other..A. granulata is an indicator of water pollution because of its sustainability in a very stressful environmental condition (Grigoryeva et al., 2019).

\section{Conclusion}

From the analysis, it was found that the blooming condition of the Aulacoseiragranulata was affected by water temperature, chlorophyll A, and dissolved oxygen. Maximum abundance was confronted during the winter season especially (December to January). The blooming condition of the plankton denotes the eutrophic status of the riverine system which may be corrected by increasing the riverine flow and reducing the anthropogenic activities in the vicinity of the river. Further studies in this direction would be of immense help in tracking the eco-health condition of the riverine system and beneficial for the greater perspective of stakeholders depending on this system.

\section{Declarations}

\section{Acknowledgment}


The authors acknowledge the National Mission for Clean Ganga (NMCG), Ministry of Water Resource, River Development and Ganga Rejuvenation, Government of India for providing the financial support to conduct the study. Thanks are also due to all the local fishers who have given their co-operation in the sampling and study process

\subsection{Funding}

The funding for the mentioned research is made by National Mission For Clean Ganga, Ministry of Jal Shakti, Government of India

\subsection{Competing Interest}

Authors declare no conflict of Interest.

\subsection{Availability of Data and Materials}

Not Applicable

\subsection{Author's Contribution}

TRM has done sampling, and MS preparation, NKT has done water sample analysyis, statistical analysis, MS preparation, SK has helped in MS preparation, AR has helped statistical data interpitation and Sampling, RKM has helped in MS correction, SB has helped in MS preparation, SR has helped in Ms preparation, SDG has helped in reverine sampling, MB has prepared the GIS map, MHR has helped in MS preparation, HSS has helped in MS preparation, BKD has conceptualized and Investigated the work. All authors read and approved the final manuscript

\subsection{Ethical Approval}

The above mentioned work is carriedout by following the ethical guidelines prescribed by Institute Ethical Commite ICAR-CIFRI.

\subsection{Consent to Participate}

Not Applicable

\subsection{Consent for Publication}

All the authors provide consent for publication.

\section{References}

1. Al-Husseini KH, Alsalman AIM (2020) Quarterly variation and their impact on phytoplankton dynamics in the Gharraf River environment in southern Iraq. Plant Archives 20:1354-1360

2. Alpha American Public Health Association (2017) Standard methods for the examination of water and wastewater. Washington, Dc, USA. Chapter 2000 pp, 1-105

3. Ambwani K, Sahni A, Kar RK, Dutta D (2003) Oldest known non-marine diatoms (Aulacoseira) from the uppermost Cretaceous Deccan Intertrappean beds and Lameta Formation of India. Rev Micropaleontol 46:67-71

4. Atique U, An KG (2019) Reservoir Water Quality Assessment Based on Chemical Parameters and the Chlorophyll Dynamics in Relation to Nutrient Regime. Polish Journal of Environmental Studies 28

5. Atique U, An KG (2020) Landscape heterogeneity impacts water chemistry, nutrient regime, organic matter, and chlorophyll dynamics in agricultural reservoirs. Ecol Ind 110:105813

6. Ayoade AA, Agarwal NK, Chandola-Saklani A (2009) Changes in physicochemical features and plankton of two regulated high altitude rivers Garhwal Himalaya, India. Eur J Sci Res 27:77-92

7. Bargu S, White JR, Li C, Czubakowski J, Fulweiler RW (2011) Effects of freshwater input on nutrient loading, phytoplankton biomass, and cyanotoxin production in an oligohaline estuarine lake. Hydrobiologia 661:377-389

8. Bedoshvili YD, Bondarenko NA, Sakirko MV, Khanayev IV, Likhoshway YV (2007) The change in the length of colonies of the planktonic diatom Aulacoseira baicalensis in various stages of the annual cycle in Lake Baikal. Hydrobiol J 43:79-86

9. Bicudo DC, Tremarin PI, Almeida PD, Zorzal-Almeida S, Wengrat S, Faustino SB, Costa LF, Bartozek EC, Rocha AC, Bicudo CE, Morales EA (2016) Ecology and distribution of Aulacoseira species (Bacillariophyta) in tropical reservoirs from Brazil. Diatom Res 31:199-215

10. Blum JL (1957) An ecological study of the algae of the Saline River. Michigan Hydrobiologia 9:361-408

11. Chakraborty B, Roy S, Bera A, Adhikary PP, Bera B, Sengupta D, Bhunia GS, Shit PK (2021) Cleaning the river Damodar (India): impact of COVID-19 lockdown on water quality and future rejuvenation strategies. Environment, Development, and Sustainability 3: 1-15

12. Cox EJ, Cox EJ (1996) Identification of freshwater diatoms from live material 1: 1-158

13. Cupp EE (1943) Marine plankton diatoms of the west coast of North America

14. Dimri D, Daverey A, Kumar A, Sharma A (2021) Monitoring water quality of River Ganga using multivariate techniques and WQI (Water Quality Index) in Western Himalayan region of Uttarakhand, India. Environmental Nanotechnology, Monitoring \& Management 15: 100375 
15. Dodds WK, Jones JR, Welch EB (1998) Suggested classification of stream trophic state: distributions of temperate stream types by chlorophyll, total nitrogen, and phosphorus. Water Res 32:1455-1462

16. Grigoryeva IL, Komissarov AB, Kuzovlev VV, Chekmareva EA (2019) Influence of thermal pollution on the ecological conditions in cooling reservoirs. Water Resour 46:S101-S109

17. Guinder VA, Malits A, Ferronato C, Krock B, Garzón-Cardona J, Martínez A (2020) Microbial plankton configuration in the epipelagic realm from the Beagle Channel to the Burdwood Bank, a Marine Protected Area in Sub-Antarctic waters. Plos one 15:e0233156

18. Guiry MD, Guiry GM (2014) AlgaeBase.[Online]. National University of Ireland, Galway

19. Guiry MD, \&Guiry GM (2021) AlgaeBase. World-wide electronic publication, National University of Ireland, Galway. http://www.algaebase.org; searched on 03 July 2021

20. Ha K, Jang MH, Joo GJ (2003) Winter Stephanodiscus bloom development in the Nakdong River regulated by an estuary dam and tributaries. Hydrobiologia 506:221-227

21. Herawati EY, Khasanah RI, Ambarwati M, Sofarini D (2020) The effect of hydro-oceanographic factors on the community structure of plankton in natural and artificial coral reefs in Paiton waters, 13. Aquaculture, Aquarium, Conservation \& Legislation, pp 71-85

22. Hong JW, Lee SD, Lee K, Hong J (2020) Seasonal variations in the surface energy and CO2 flux over a high-rise, high-population, residential urban area in the East Asian monsoon region. Int J Climatol 40:4384-4407

23. Hötzel G, Croome R (1996) Population dynamics of Aulacoseira granulata (Ehr.) Simonsen (Bacillariophyceae, Centrales), the dominant alga in the Murray River. Australia Arch Hydrobiol 136:191-215

24. Jewson DH (1992) Size reduction, reproductive strategy and the life cycle of a centric diatom. Philosophical Transactions of the Royal Society of London Series B: Biological Sciences 336:191-213

25. Jia XH, Jiang WX, Li FQ, Tang T, Duan SG, Cai QH (2009) Impacts of large hydropower stations on benthic algal communities. Ying yong sheng tai xue bao $=$ The. J Appl Ecol 20:1731-1738

26. Joung SH, Park HK, Lee HJ, Lee SH (2013) Effect of climate change for diatom bloom at winter and spring season in Mulgeum Station of the Nakdong River, South Korea. Journal of Korean Society on Water Environment 29:155-164

27. Kanaya G, Kikuchi E (2008) Spatial changes in a macrozoobenthic community along environmental gradients in a shallow brackish lagoon facing Sendai Bay, Japan. Estuarine, Coastal and Shelf Science 78: 674-684

28. Kilham SS, Kilham P (1975) Melosira granulata (Ehr.) Ralfs: morphology and ecology of a cosmopolitan freshwater diatom: With 2 figures and 1 table in the text. Internationale Vereinigung für theoretische angewandte Limnologie: Verhandlungen 19:2716-2721

29. Kulikovskiy M, Glushchenko AM, Kuznetsova IV, \&Genkal SI (2016) Identification book of diatoms from Russia. pp. [1]-804, 165 pls. Filigran, Yaroslavl. [in Russian]

30. Lakshminarayana JSS (1965) Studies on the phytoplankton of the River Ganges, Varanasi, India, Part II "The seasonal growth and succession of the plankton algae in the River Ganges". Hydrobiologia 25:138-165

31. Lee HJ, An KG (2009) The Development and Application of Multi-metric Water Quality Assessment Model for Reservoir Managements in Korea. Korean Journal of Ecology Environment 42:242-252

32. Margalef R (1983) Limnología. Omega, Barcelona, 1001 pp

33. Matta G, Kumar A, Nayak A, Kumar P, Kumar A, Tiwari AK (2020) Water quality and planktonic composition of river Henwal (India) using comprehensive pollution index and biotic-indices. Transactions of the Indian National Academy of Engineering 5:541-553

34. Mayama S (1991) Taxonomic revisions to the differentiating diatom groups for water quality evaluation and some comments for taxa with new designations. Nova Hedwigia 52

35. Moro RS (1991) Morphology of Aulacoseira granulata (Ehrenberg) var. australiensis (Grunow) nov. comb. unser light microscopy. Arquivos de Biologia e Tecnologia 34:353-359

36. Nwankwegu AS, Li Y, Huang Y, Wei J, Norgbey E, Sarpong L, Wang K (2019) Harmful algal blooms under changing climate and constantly increasing anthropogenic actions: the review of management implications. 3 Biotech 9:1-19

37. Pandey U, Pandey J, Singh AV, Mishra A (2017) Anthropogenic drivers shift diatom dominance-diversity relationships and transparent exopolymeric particles production in river Ganga: implication for natural cleaning of river water. Current Science, 959-964

38. Parmar TK, Rawtani D, Agrawal YK (2016) Bioindicators: the natural indicator of environmental pollution. Frontiers in life science 9:110-118

39. Pathak V, Tyagi RK (2010) Riverine ecology and fisheries visa-vis hydrodynamic alterations: impacts and remedial measures. CIFRI, bulletin, (161)

40. Pham L (2017) Comparison between Water Quality Index (WQI) and biological indices, based on planktonic diatom for water quality assessment in the Dong Nai River, Vietnam. Pollution 3:311-323

41. Poister D, Kurth A, Farrell A, Gray S (2012) Seasonality of Aulacoseira ambigua abundance and filament length: biogeochemical implications. Plankton Benthos Research 7:55-63

42. Poister D, Schaefer A, Baert A, Tracey J, Richards K (2015) Stimulated rejuvenation of dormant Aulacoseira granulata (Bacillariophyta) by Gloeocystis planctonica (Chlorophyta) in a eutrophic river. J Phycol 51:332-342

43. Popovic NZ, Duknic JA, Atlagic JZ, Rakovic MJ, Marinkovic NS, Tubic BP, Paunovic MM (2016) Application of the water pollution index in the assessment of the ecological status of rivers: a case study of the Sava River, Serbia. Acta Zoologica Bulgarica 68:97-102 
44. Pratiwi NT (2019, July) Community Structure of Plankton at Central Kalimantan Peat Swamp Area. In IOP Conference Series: Earth and Environmental Science 298: 012003

45. Quincey DJ (2017) The Himalayan Climate and Water Atlas. Mt Res Dev 37:155-157

46. Rimet F, Bouchez A, Montuelle B (2015) Benthic diatoms and phytoplankton to assess nutrients in a large lake: complementarity of their use in Lake Geneva (France-Switzerland). Ecological indicators 53:231-239

47. Roshith CM, Meena DK, Manna RK, Sahoo AK, Swain HS, Raman RK, Sengupta A, Das BK (2018) Phytoplankton community structure of the Gangetic (Hooghly-Matla) estuary: Status and ecological implications in relation to eco-climatic variability. Flora 240:133-143

48. Sarkar SD, Sahoo AK, Gogoi P, Raman RK, Munivenkatappa MH, Kumari K, Mohanty BP, Das (2019) Phytoplankton biomass in relation to flow dynamics: the case of a tropical river Mahanadi, India. Tropical Ecology 60:485-494

49. Sekerci Y, Petrovskii S (2018) Global warming can lead to depletion of oxygen by disrupting phytoplankton photosynthesis: a mathematical modelling approach. Geosciences 8:201

50. Shende S, Rathoure AK Physico-chemical Properties of Arai river in monsoon near Chandrapur city (maharashtra) India

51. Srivastava K, Mishra S, Verma HO, Thakur VR, Jha DN, Alam A, Das BK (2020) Time scale changes of plankton in the river Ganga at Kanpur

52. Srivastava P, Verma J, Grover S, Sardar A (2016) On the importance of diatoms as ecological indicators in river ecosystems: Areview. Indian J Plant Sci $5: 70-86$

53. Taylor JC, Harding WR, Archibald CGM (2007) An illustrated guide to some common diatom species from South Africa. Water Research Commission, Pretoria, p 225

54. Thomas MK, Aranguren-Gassis M, Kremer CT, Gould MR, Anderson K, Klausmeier CA, Litchman E (2017) Temperature-nutrient interactions exacerbate sensitivity to warming in phytoplankton. Glob Change Biol 23:3269-3280

55. Tsai YC, Chen KC, Cheng TS, Lee C, Lin SH, Tung CW (2019) Chlorophyll fluorescence analysis in diverse rice varieties reveals the positive correlation between the seedlings salt tolerance and photosynthetic efficiency. BMC Plant Biol 19:1-17

56. Wang C, Lek S, Lai Z, Tudesque L (2017) Morphology of Aulacoseira filaments as indicator of the aquatic environment in a large subtropical river: The Pearl River, China. Ecol Ind 81:325-332

57. Wang C, Wu N, Li W, Liu Q, Lai Z, Fohrer N (2020) Curved filaments of Aulacoseira complex as ecological indicators in the Pearl River, China. Ecol Ind 118:106722

58. Wang Q, Wu X, Zhao B, Qin J, Peng T (2015) Combined multivariate statistical techniques, water pollution index (WPI) and Daniel trend test methods to evaluate temporal and spatial variations and trends of water quality at Shanchong River in the Northwest Basin of Lake Fuxian, China. PloS one 10:e0118590

59. Wetz MS, Wheeler PA (2007) Release of dissolved organic matter by coastal diatoms. Limnology Oceanography 52:798-807

60. Yang JR, Basu BK, Hamilton PB, Pick FR (1997) The development of a true riverine phytoplankton assemblage along a lake-fed lowland river. - Arch Hydrobiol 140:243-260

61. Yang Q, Xie P, Shen H, Xu J, Wang P, Zhang B (2012) A novel flushing strategy for diatom bloom prevention in the lower-middle Hanjiang River. Water Res 46:2525-2534

62. You Q, Liu Y, Wang Y, Wang Q (2009) Taxonomy and distribution of diatoms in the genera Epithemia and Rhopalodia from the Xinjiang Uygur Autonomous Region, China. Nova Hedwigia 89:397-430

63. Zhang Y, Peng C, Wang Z, Zhang J, Li L, Huang S, Li D (2018) The species-specific responses of freshwater diatoms to elevated temperatures are affected by interspecific interactions. Microorganisms 6:82

64. Zheng BH, Chen ZJ, Li YY, Fohrer N, Zhang Y, Wu DY, Yan XY, Li BL (2020) Structural Characteristics and Driving Factors of the Planktonic Eukaryotic Community in the Danjiangkou Reservoir. China Water 12:3499

\section{Figures}



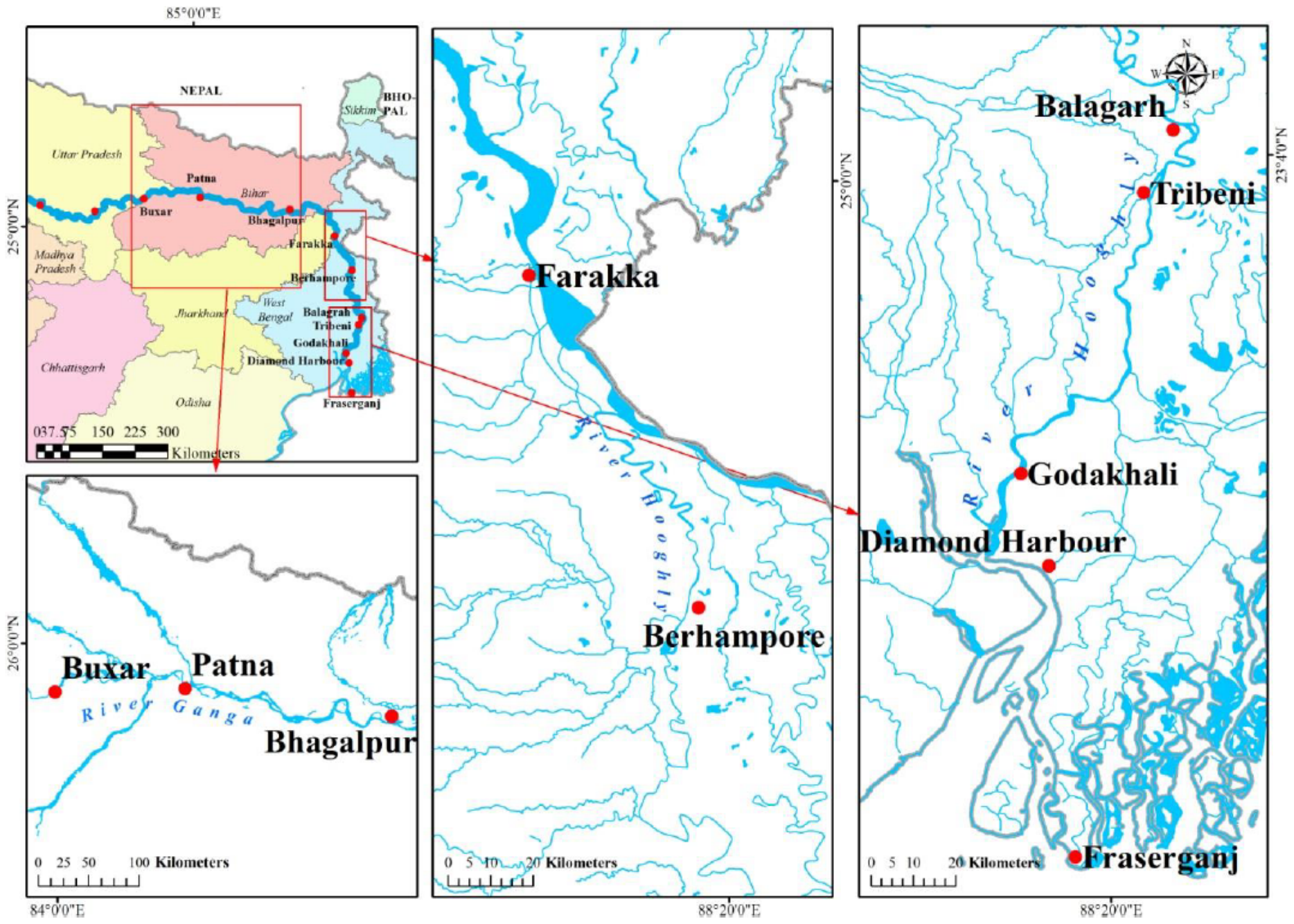

Figure 1

Study area map of the sampling sites 


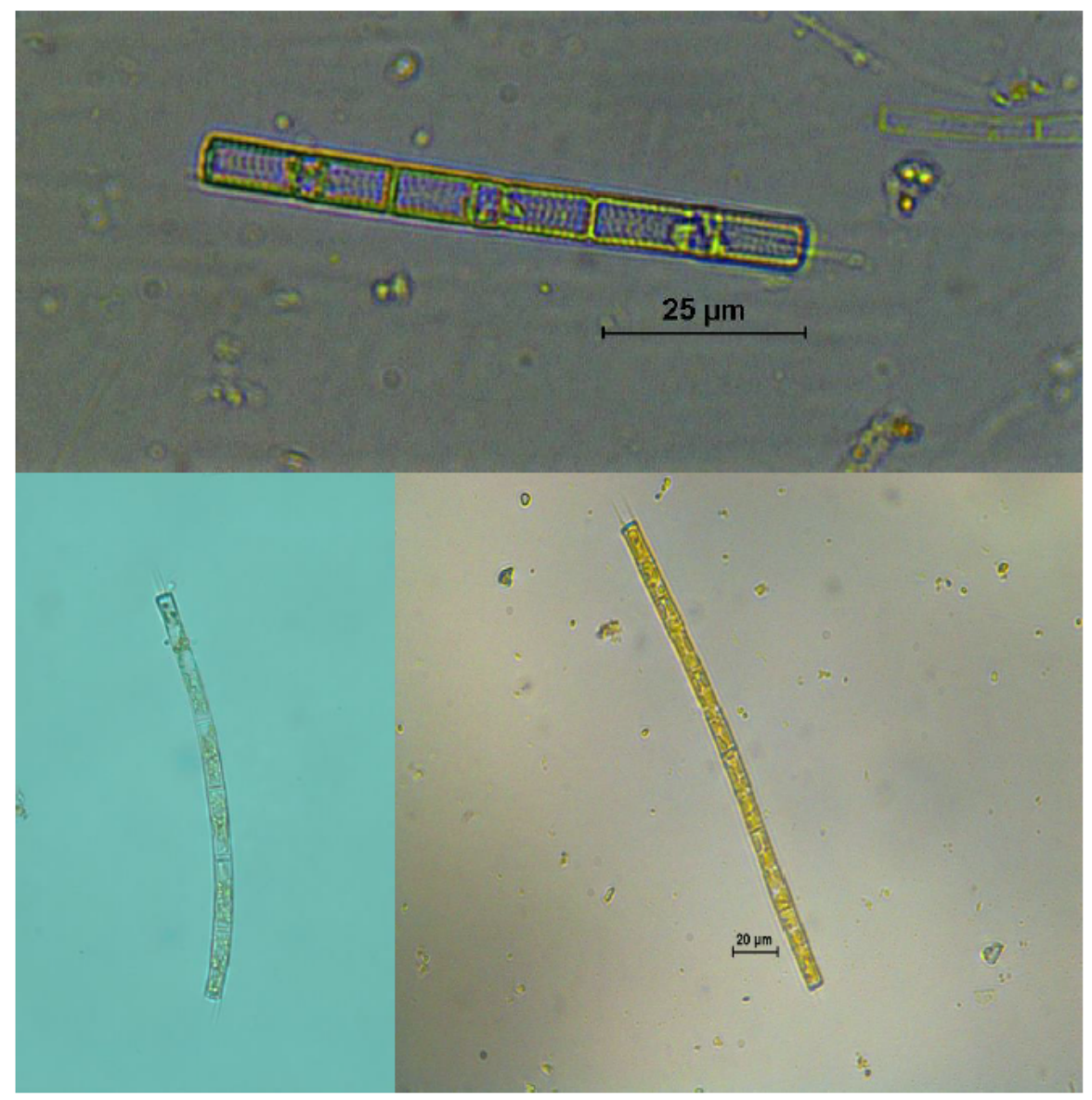

\section{Figure 2}

Aulacoseira granualata observed at different sampling sites

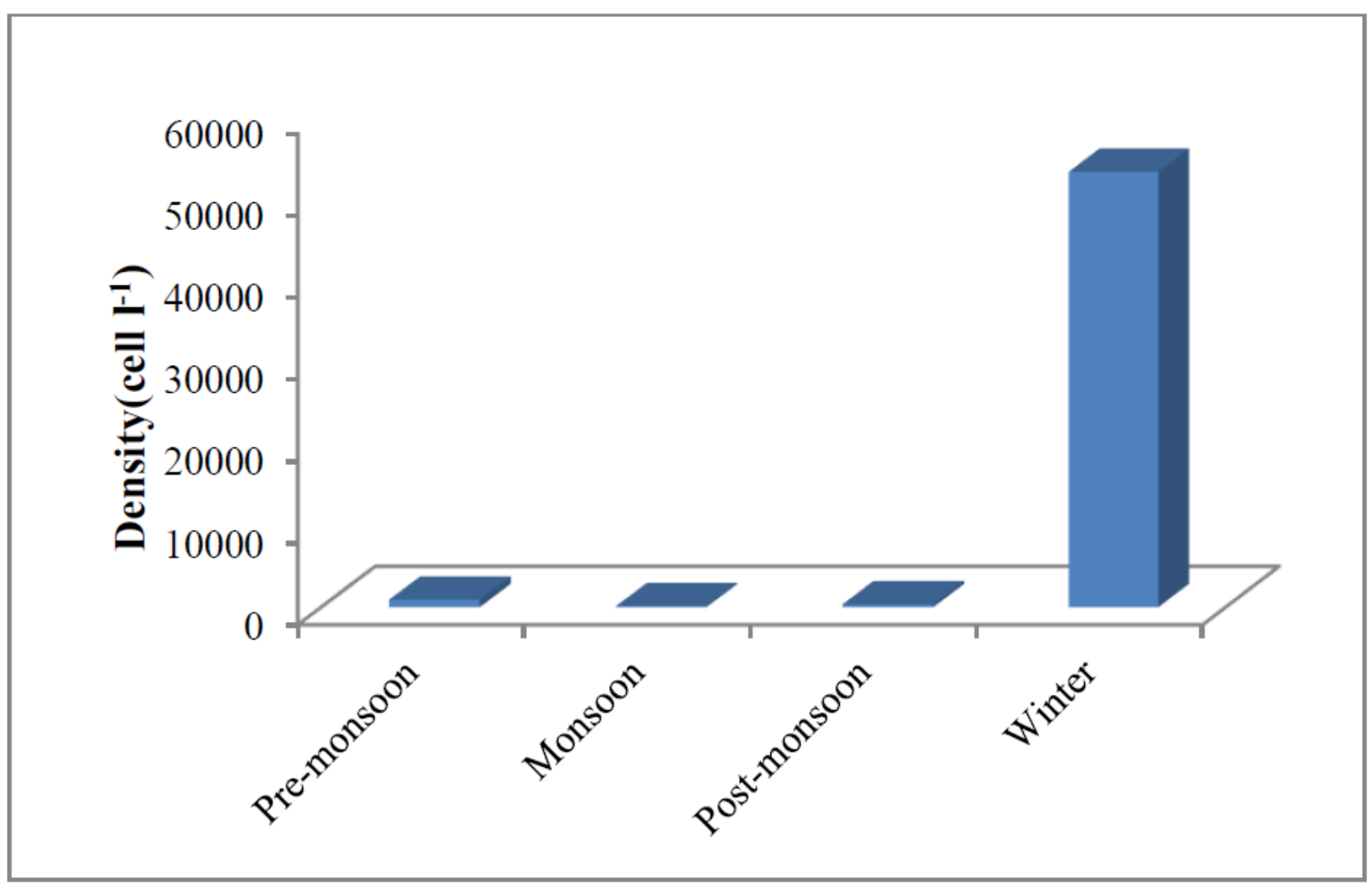

Figure 3 


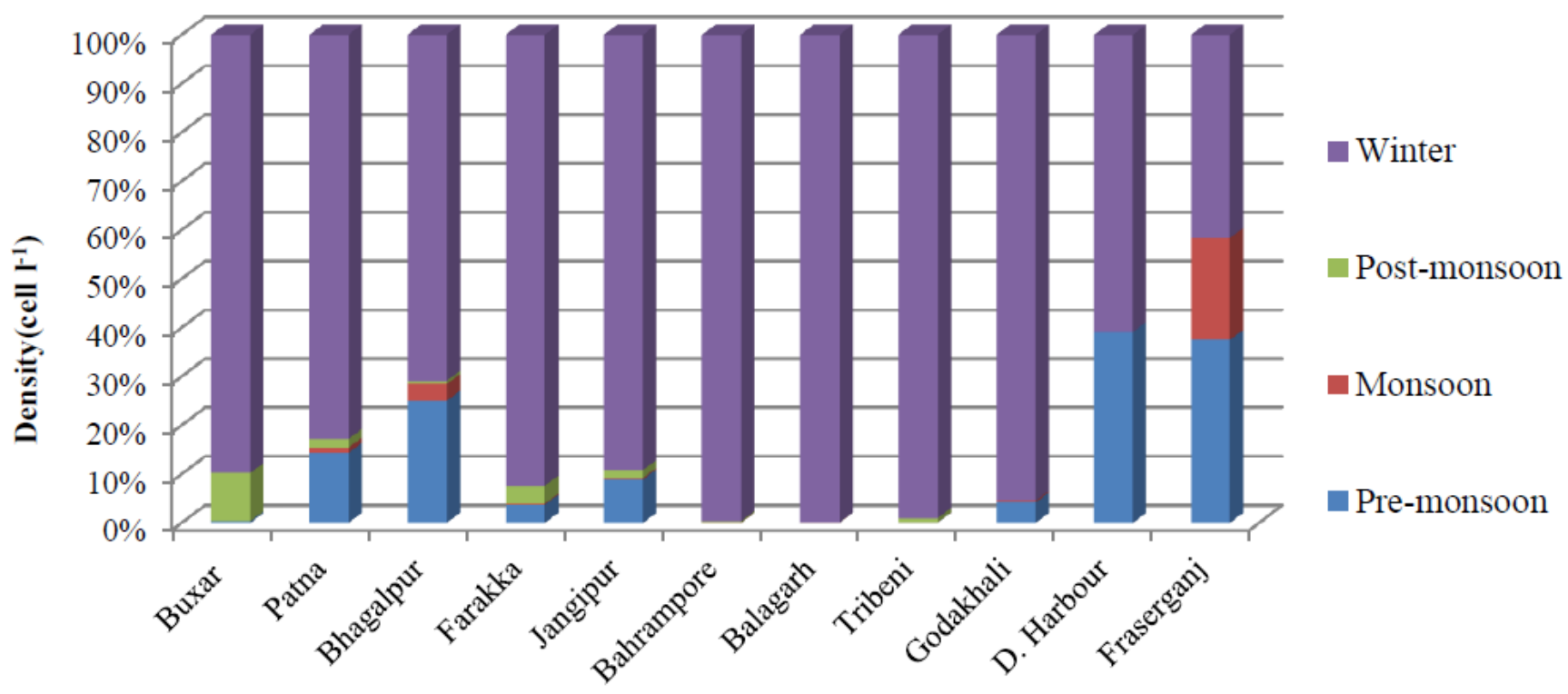

Figure 4

Seasonal variation of A.granulata at different station

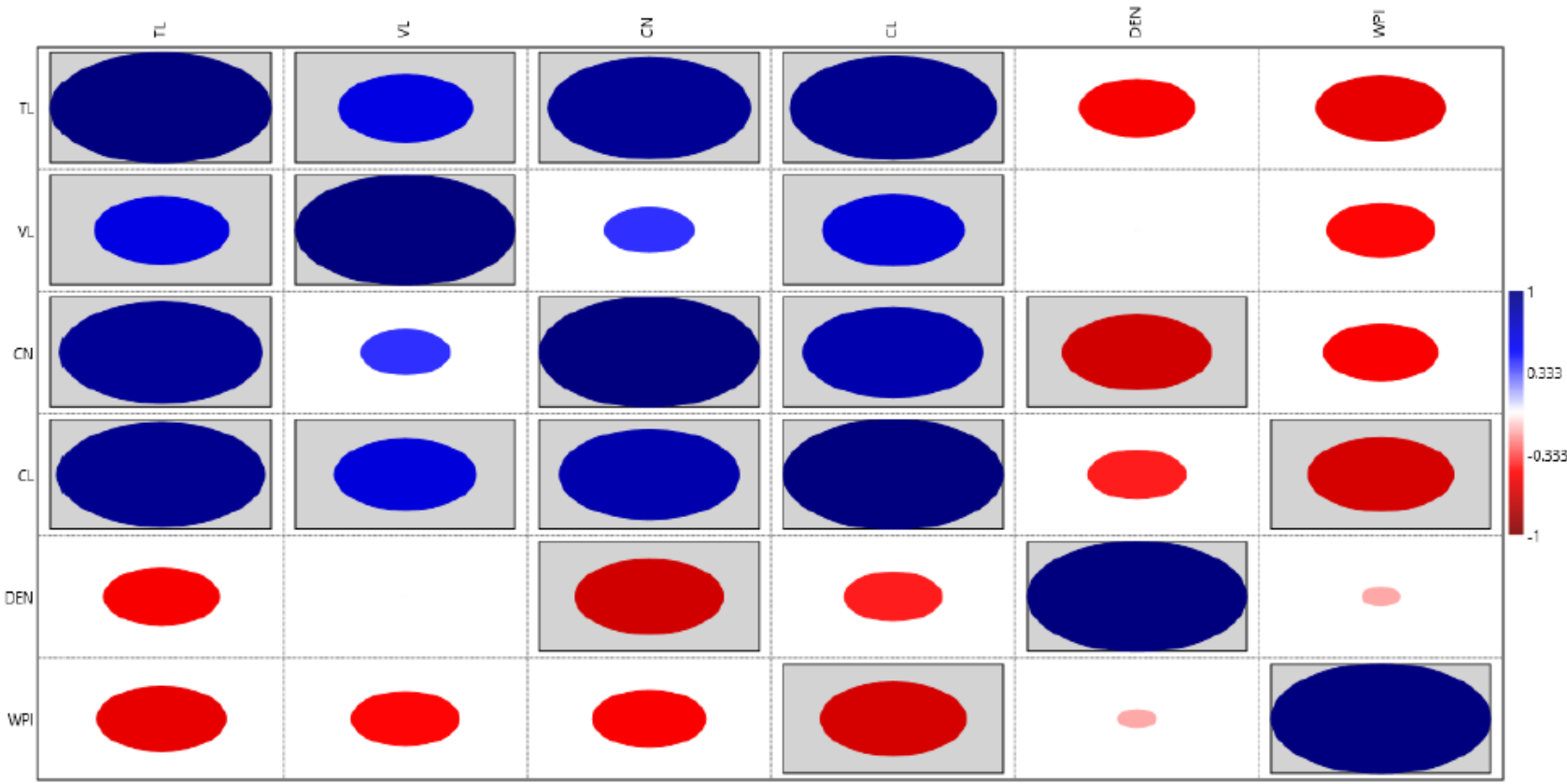

Figure 5

Showing correlation of Water Pollution Index with morphological characters (TL-Total Length, VL- Valve length, CN- Cell Number, DEN- Density, WPI- Water Pollution Index) 

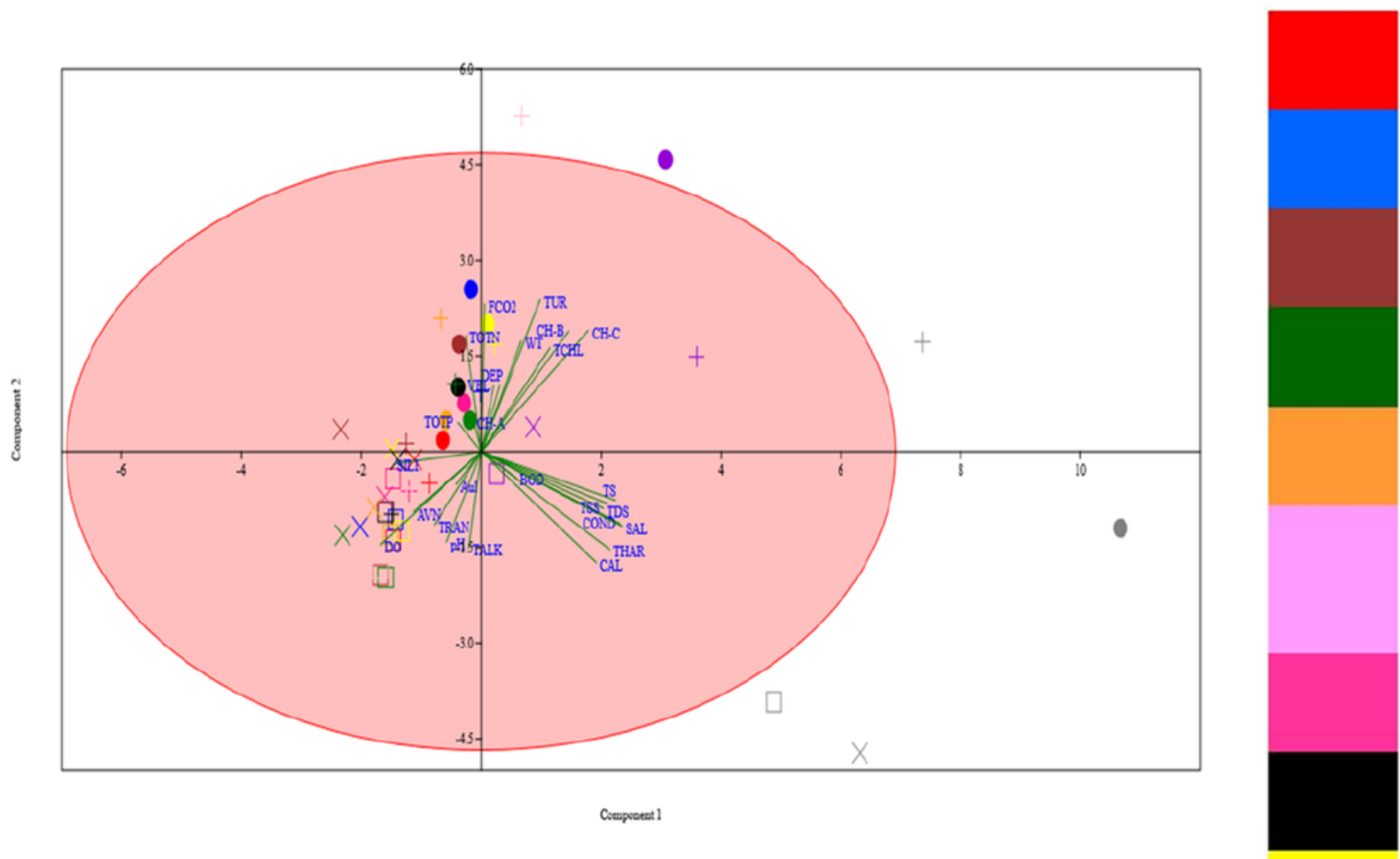

BUXAR

PATNA

BHAGALPUR

FARAKKA

JANGIPUR

BERHAMPORE

BALAGARH

TRIBENI

\begin{tabular}{|c|c|}
\hline DOT & MONSOON \\
\hline PLUS & POST MONSOON \\
\hline SQUARE & WINTER \\
\hline CROSS & PRE MONSOON \\
\hline
\end{tabular}

GODAKHALI

D. HARBOUR

FRASERGAN

Figure 6

PCA bi-plot of the different environmental parameters 


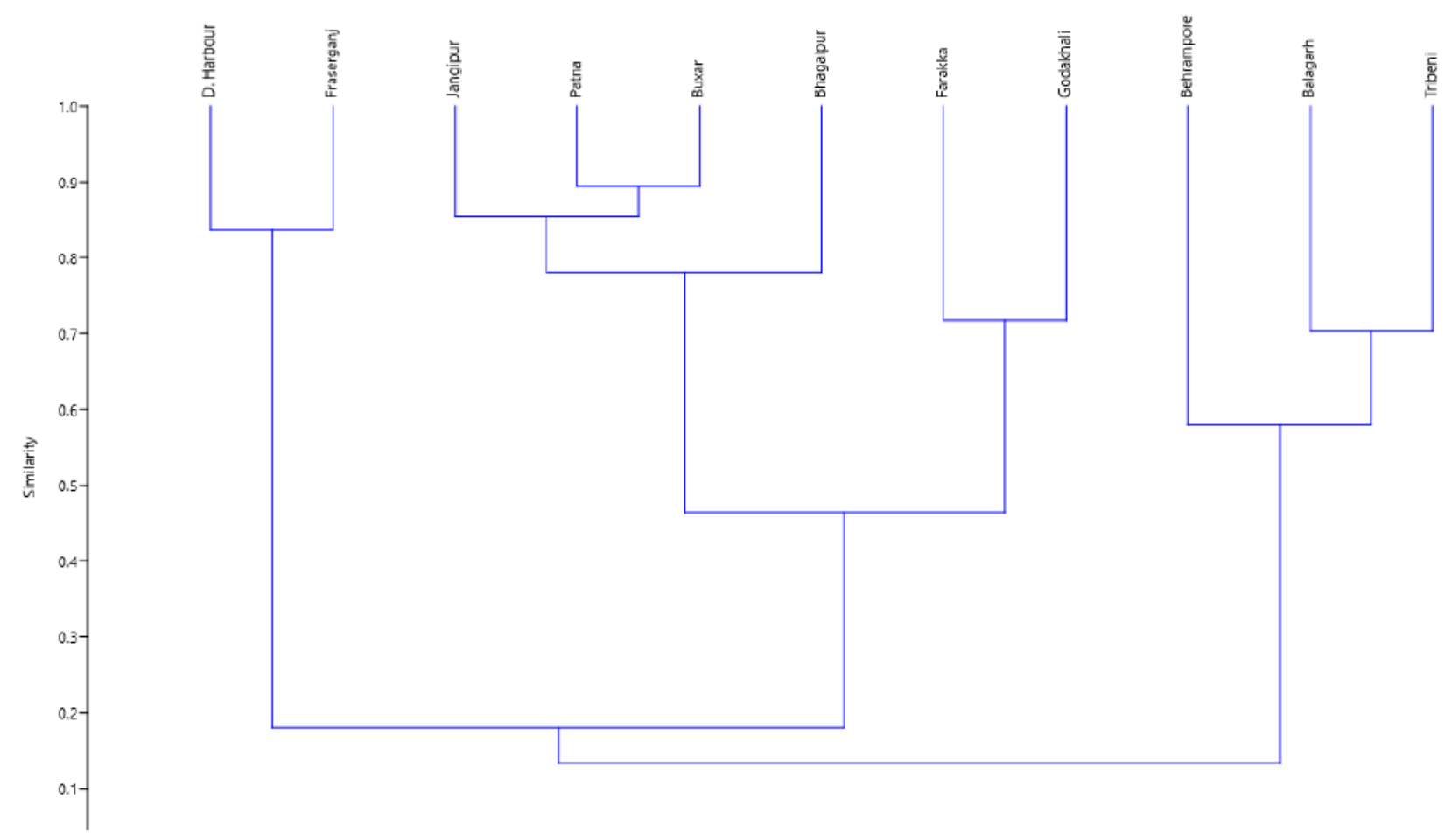

Figure 7

Bray Curtis cluster analysis of studied station 